\title{
Effects of Chinese herbal medicine B307 supplementation on muscle endurance and recovery after exhaustive swimming in ICR mice
}

\section{Tai-Yuan Chuang}

National Taiwan Normal University

Chia-Ying Lien

National Taiwan University of Physical Education and Sport

Ya-Chun Tsai

National Tsing Hua University - Nanda Campus

Kuei-Fu Lin

National Tsing Hua University - Nanda Campus

Chih-Hsiang Hsu

National Taiwan Normal University

Wen-Jhen Wu

National Taiwan Normal University

Li-Yu Su

National Taiwan Normal University

Chen-Wen Lu

National Taiwan Normal University

Chung-Hsin Wu ( $\nabla$ megawu@ntnu.edu.tw)

National Taiwan University Department of Life Science https://orcid.org/0000-0002-4470-2448

\section{Research}

Keywords: Chinese herbal medicine, Exhaustive swimming, Muscular endurance 37 Oxidative stress, Inflammation, Gastrocnemius muscles, ICR mice

Posted Date: February 21st, 2020

DOI: https://doi.org/10.21203/rs.2.24182/v1

License: (c) (1) This work is licensed under a Creative Commons Attribution 4.0 International License.

Read Full License 


\section{Effects of Chinese herbal medicine B307 supplementation on muscle 2 endurance and recovery after exhaustive swimming in ICR mice}

3 Authors: Tai-Yuan Chuang 1,2, Chia-Ying Lien2, Ya-Chun Tsai³, Kuei-Fu Lin ${ }^{3}$, Chih-Hsiang Hsu', Wan-Jhen Wu', Li-Yu Su ', Chen-Wen Lu', and Chung-Hsin Wul,*

Affiliation: ' School of Life Science, National Taiwan Normal University, Taipei City, Taiwan 2 Department of Athletics, National Taiwan University, Taipei City, Taiwan 3 Department of Physical Education, National Tsing Hua University, Hsinchu City, Taiwan

$11{ }^{*}$ Corresponding author:

12 Chung-Hsin Wu

13 Professor of Department of Life Sciences, National Taiwan Normal University

14 Tel: +886-2-77346363, Fax: +886-2-29312904, E-mail: megawu@ntnu.edu.tw

15 ADD: School of Life Science, No. 88, Sec 4, Ting-Chow Rd, Taipei, Taiwan, 1 16, R.O.C. 


\section{ABSTRACT:}

18 Background: Exhaustive exercise may damage muscles due to oxidative stress and

19 inflammation and cause muscle fatigue and soreness. The study investigated the

20 effects of Chinese herbal medicine (CHM) B307 supplementation on muscle

21 endurance and recovery after exhaustive swimming (ES) in ICR mice.

22 Methods: All mice were subjected to ES in the form of a forced swimming test. Thirty-two

23 male ICR mice were randomly divided into 4 groups: Sham + ES, CHM B307

24 pretreatment + ES (Pre+ES), CHM B307 posttreatment + ES (Post+ES), and CHM B307

25 dual treatment + ES (Dual+ES). Using immunohistochemistry, chemiluminescence, and

26 biochemical analysis, muscular endurance, fatigue, oxidative stress, inflammation, and

27 damage were examined and compared among the groups.

28 Results: Our results revealed that the Pre+ES and Dual+ES groups had remarkably better 29 muscular endurance during ES than the Sham+ES and Post+ES groups. In addition, the 30 Pre+ES, Post+ES, and Dual+ES groups had significantly alleviated fatigue, oxidative 31 stress, inflammation, and damage after ES compared with the Sham+ES group.

32 Conclusion: CHM B307 can be a protective supplement against damage from 33 exhaustive exercise because of its antifatigue, antioxidation, anti-inflammation, and 34 antidamage functions.

Keywords: Chinese herbal medicine, Exhaustive swimming, Muscular endurance Oxidative stress, Inflammation, Gastrocnemius muscles, ICR mice 


\section{Introduction}

40 Humans who participate in acute endurance exercise often increase their oxygen

41 consumption, which produces reactive oxygen species (ROS) and causes muscle

42 damage [1-3]. ROS may play a crucial role in body defense systems [4], but excessive

43 ROS contributes to diseases and conditions such as diabetes, cancer, cardiovascular 44 disease, and neurological disorders, even affecting aging processes [5, 6]. Endogenous

45 antioxidant capacity also reportedly increases following exercise, which can partially 46 attenuate oxidative stress by scavenging ROS [7-10]. Even though individuals can

47 remove ROS from their bodies, intense or prolonged exercise generates a considerable 48 number of ROS, far more than our antioxidant capacity [11]. As a result, excessive ROS

49 causes oxidative stress because of an imbalance between oxidants and antioxidants in 50 favor of oxidants. Many sports science studies have indicated that skeletal muscle 51 fibers exposed to a high concentration of ROS may lead to impaired muscle force 52 production and fatigue [12-14].

53 Athletes who engage in exhaustive exercise with limited recovery periods may see 54 their athletic performance decline and muscle fatigability increase subsequently [15].

55 Excessive exercise can affect the balance of hormone adjustment and cause 56 oxidative stress and subsequent cell membrane damage in both male and female rats [16]. To examine physiological damage induced by exhaustive exercise, forced swimming is widely used in studies of exhaustive exercise testing in rats [17]. In forced swim tests, exercise intensity can be indirectly graded by the addition of a weight load attached to the body or tail to decrease the time until exhaustion [18]. Thus, forced

62 physiological damage.

Unlike health-promoting exercise, athletes must undergo severe training daily to

64 improve their athletic performance; therefore, identifying an appropriate sports

65 supplement to protect muscles against oxidative stress and inflammation is essential. It

66 was reported that Danggui Buxue Tang has a favorable antifatigue effect in male mice 67 after forced swimming [19]. Thus, Chinese herbal medicines (CHMs) may provide 
68 natural food sources of sports supplements for achieving antifatigue effects. To our 69 knowledge, however, the antiantioxidant, anti-inflammatory, and muscle-relieving 70 effects of $\mathrm{CHMs}$ after forced swim tests have rarely been elucidated. As we have 71 previously suggested, CHM B307 can effectively enhance muscle strength in mice [20,

72 21]. B307 may be an appropriate sports supplement to protect muscle against

73 oxidative stress and inflammation for athletes training under a high exercise intensity.

74 Therefore, the purpose of this study was to examine the antiantioxidant,

75 anti-inflammatory, and muscle-relieving effects of CHM B307 in Institute of Cancer

76 Research (ICR) mice after an exhaustive swimming (ES) test.

78 Materials and Methods

79 Animal preparation

80 Thirty-two male ICR mice were purchased from BioLASCO Taiwan Yi-Lan Breeding

81 Center (fully accredited by AAALAC International). In accordance with the Institutional

82 Guidelines of the Animal Care and Use Committee of National Taiwan Normal

83 University (NTNU), the mice were maintained in the animal facility of NTNU under

84 specific pathogen-free conditions. All animal experiments were approved by the

85 Institutional Animal Care and Use Committee of our university (Protocol number: NTNU

86 Animal Experiments No. 108011). All the mice were housed in an environment with a

87 constant temperature of $22^{\circ} \mathrm{C} \pm 2{ }^{\circ} \mathrm{C}$ and subjected to a 12-h light/dark cycle, and the 88 mice had ad libitum access to water and food.

\section{Study design}

91 The study assessed the effects of Chinese herbal medicine (CHM) B307

92 supplementation on muscle endurance and recovery after ES in ICR mice. As

93 suggested in Figure 1, all mice were subjected to forced ES executed under a forced

94 swimming test. A total of 32 male ICR mice were randomly divided into 4 groups: Sham

$95+$ ES, CHM B307 pretreatment + ES (Pre+ES), CHM B307 posttreatment + ES (Post+ES),

96 and CHM B307 dual treatment + ES (Dual+ES). The Pre+ES mice were orally given B307 
97 treatment before ES, Post+ES mice were orally given B307 treatment after ES, Dual+ES

98 mice were orally given B307 treatment both before and after ES, and the Sham+ES

99 group was given a normal diet and drinking water before and after ES. The experiment 100 was approved by the Institutional Animal Care and Use Committee of NTNU (protocol 101 number NTNU Animal Experiments No. 108011).

102

103 ES experiment design

104 As suggested in [22], a weight-loaded ES procedure was carried out in a swimming 105 tank $\left(50 \times 50 \times 50 \mathrm{~cm}^{3}\right)$ with 30 - $\mathrm{cm}$-deep water maintained at $25 \pm 3^{\circ} \mathrm{C}$ for $1 \mathrm{~h}$ after the 106 oral administration of CHM B307. Then mice were removed from the pool, dried with a 107 paper towel, and returned to their original cages. The pool water was replaced after 108 each session. ES was determined by observing the loss of coordinated movements and 109 failure to swim. ES time was recorded immediately when mice were completely 110 exhausted and failed to return to the surface to breathe within $5 \mathrm{~s}$.

\section{Oral treatment of CHM B307}

113 CHM B307 is a commercially available nutritional supplement (Sun-Ten Pharmaceutical 114 Company, New Taipei City, Taiwan). The Pre+ES, Post+ES, and Dual+ES groups were 115 orally treated with CHM B307 extract. We used a gavage tube to orally administer CHM 116 B307 extract (50 mg/mL; the pH value was approximately 7.0) and its vehicle (dimethyl 117 sulfoxide). Doses were adjusted according to the individual weight of the mouse. The 118 feeding frequency was 4 times for the Pre+ES and Post+ES mice and 8 times for the 119 Dual+ES mice at intervals of 12 hours. The dosage and administration of CHM B307 120 extract were much lower than the median lethal dose (LD $\left.{ }_{50}\right)$. The sham group was 121 orally treated with the vehicle.

122

\section{Chromatographic fingerprint analysis of CHM B307}

124 All CHM B307 chemicals were solubilized in distilled $\mathrm{H}_{2} \mathrm{O} / \mathrm{MeOH}$ and then analyzed 125 using cetonitrile of high-performance liquid chromatography grade (Burdick \& Jackson, 
126 Gyeonggi-do, Korea) and methanol (Avantor, Center Valley, PA, USA). Purified water 127 from a Milli-Q water purification system (EMD Millipore, Billerica, MA, USA) was used to 128 dissolve the ingredients.

129

130

\section{Urine and blood tests}

After the final ES procedure, mice were immediately sacrificed under mild anesthesia. Urine was collected from the bladder, and blood was collected from the abdominal aorta into centrifuge tubes using a heparinized syringe. The urine samples were analyzed using 10 parameter urinalysis reagent strips for urine tests (Medisave Ltd., Weymouth, UK). The blood samples were analyzed using a blood smear, as is common practice to evaluate the health status of an animal. The blood samples were taken in a blood sampling tube with heparin (Terumo Inc., Tokyo, Japan) and centrifuged for 10 min at 1,000 $\times \mathrm{g}$ at $4{ }^{\circ} \mathrm{C}$ to separate the plasma, which was stored at $-80{ }^{\circ} \mathrm{C}$ until analysis. The plasma samples were analyzed for lactate acid using an assay kit (Cayman Chemical, MI, USA). To determine blood ROS levels, lucigenin- and Iuminol-amplified chemiluminescence $(\mathrm{CL})$ methods were used to measure $\mathrm{O} 2 \cdot$ - and $\mathrm{H}_{2} \mathrm{O}_{2}$ activity. The lucigenin-enhanced $\mathrm{CL}$ method is reliable for ROS assays. A heparinized sample $(0.2 \mathrm{~mL})$ of whole blood was taken from the left femoral artery of each mouse. ROS blood levels were measured using a CL analyzer (CLA-ID3 chemiluminescence analyzer; Tohoku Electronic Industrial Co., Ltd., Sendai, Japan) after the addition of $1.0 \mathrm{~mL}$ of $0.1 \mathrm{mM}$ lucigenin in phosphate-buffered saline $(\mathrm{pH} \mathrm{7.4)}$ to 147 the samples. The assay was duplicated for each sample, and total CL counts in $600 \mathrm{~s}$ 148 were calculated by integrating the area under the curve.

\section{Muscle immunohistochemistry analysis}

151 ICR mice were anesthetized and then cardiac-perfused with phosphate-buffered 152 saline containing 4\% formaldehyde (EM grade glutaraldehyde solution, Sigma-Aldrich 153 St. Louis, MU, United States ). Gastrocnemius muscle tissue was then removed and fixed 154 with $4 \%$ formaldehyde (EM grade). The muscle specimens were embedded in paraffin 
155 and cut into tissue sections with a thickness of $5 \mu \mathrm{m}$. The tissue sections were mounted 156 on slides for histological and muscle immunohistochemistry (IHC) analysis. Using the 157 heat-induced epitope retrieval method, the sections were separately stained at room 158 temperature for $1 \mathrm{~h}$ with antibodies of superoxide dismutase 2 (SOD2; Cat. \# 13141, Cell 159 Signaling Technology Inc., Danvers, MA, USA), tumor necrosis factor alpha (TNF-a) (Cat. 160 \# 1 1948, Cell Signaling Technology Inc.), nuclear factor kappa-light-chain-enhancer of 161 activated B cells (NFKB) (Cat. \#3034, Cell Signaling Technology Inc.), and vinculin (Cat. 162 \# 13901, Cell Signaling Technology Inc.) immunostaining controls for each antibody.

163 Serial 5 - $\mu \mathrm{m}$ cross-sections were treated with the unanimous staining protocol.

164 Immunostaining detection was executed using incubation with biotinylated secondary 165 antibodies (NovolinkTM polymer detection system I) at room temperature for $30 \mathrm{~min}$

166 and then by incubation with avidin-biotin-HRP complex (NovolinkTM polymer 167 detection system I) for an additional $30 \mathrm{~min}$. Immunostaining visualization was 168 performed with DAB Chromogen (NovolinkTM polymer detection system I) and 169 counterstained with hematoxylin (NovolinkTM polymer detection system I) following the 170 supplier's protocol.

171

\section{Muscle Western blot analysis}

173 The removed gastrocnemius muscle tissue was kept in a buffer solution to maintain its $174 \mathrm{pH}$ for a Western blot analysis. Skeletal muscle protein was subjected to sodium 175 dodecyl sulfate-polyacrylamide gel electrophoresis and transferred to a 176 polyvinylidene difluoride membrane. We used SOD2 antibodies (Cat. \# 13141, Cell 177 Signaling Technology Inc.) to identify expression levels of anti-oxidative proteins in the 178 skeletal muscle tissue by means of a horseradish peroxidase-linked secondary antibody. 179 In addition, Enhanced chemiluminescence Western blotting detection reagents (GE 180 Healthcare Life Sciences, Piscataway, NJ, USA) were used to make immunoreactive 181 bands perceptible. An ImageQuant LAS-4000 biomolecular imager (GE Healthcare Life 182 Sciences) was used to detect chemiluminescence. Image J software (version 1.48t, 183 Wayne Rasnabd, USA) was used to count densitometric assessments of the bands. 


\section{Statistical analysis}

186 The data in Figures 3, 4, and 6 were obtained from at least 3 independent experiments.

187 Values for the data were expressed as mean \pm standard error of the mean (SEM).

188 Differences among the ICR mice groups were evaluated using a two-way analysis of 189 variance (ANOVA). If a significant F-value was observed, the Student-Newman-Keuls 190 multiple comparisons posttest was conducted to determine where differences existed. $191 P$ values of $<0.05$ were considered significant.

\section{Results}

\section{Chromatographic fingerprint of CHM B307}

195 Chromatographic fingerprint analysis of CHM B307 are shown in Figure 2. Ginseng 196 (panax ginseng Radix), schizandra (schizandrae Fructus), ophiopogon (ophiopogonis 197 Tuber), and danshen (salviae miltiorrhizae Radix) are the main herbal ingredients in 198 CHM B307. A bioactive marker substance for ginseng Radix is ginsenoside Rbl; marker 199 substances for schizandrae Fructus are schizandrin and gomisin A; a marker substance 200 for ophiopogonis Tuber is methylophiopogonanone B; and marker substances for 201 salviae militiorrhizae Radix are rosmarinic acid, salvianolic acid B, and tanshinone IIA. 202

Effects of oral CHM B307 treatment on the body weight and ES time of mice

CHM B307 is related to cardiac function and skeletal muscle strength in mice [20, 21]. We sought to determine whether any change in muscle endurance to ES occurred with oral CHM B307 treatment. We measured the body weight and ES time of ICR mice after oral CHM B307 treatment. After ES, no significant difference was observed in body weight in any group ( $p>0.05$, Fig. 3A). However, as presented in Figure 3B, the Pre+ES and Dual+ES groups had ES times $250 \%$ longer than those of the Sham+ES and Post+ES groups $(\rho<0.01$, Fig. 3B). The results suggest that CHM B307 pretreatment promoted 
213 Effects of oral CHM B307 treatment on pathological factors in urine and blood after ES

214 We examined the effects of oral CHM B307 treatment in inflammatory factors in urine

215 and blood. Figure 4A presents a comparison of leukocyte counts in urine among the 216 groups. Leukocyte counts in the urine of the Sham+ES mice were significantly increased 217 after ES, whereas the leukocyte counts of the Pre+ES, Post+ES, and Dual+ES mice were 218 significantly decreased $(P<0.01$, Fig. 4A). We also compared neutrophil counts in 219 blood samples (Fig. 4Ba). Compared with normal mice, neutrophil counts for the 220 Sham+ES mice were significantly increased after ES, and neutrophil counts for the 221 Pre+ES, Post+ES, and Dual+ES mice were significantly decreased ( $P<0.01$, Fig. 4Ba). 222 To examine the effects of oral CHM B307 treatment on muscle fatigue, we 223 compared lactic acid levels in the blood of the mice (Fig. 4Bb). Lactic acid levels of the 224 Sham+ES mice were significantly increased after ES, whereas those of the other groups 225 were significantly decreased $(p<0.01)$.

Effect of oral CHM B307 treatment on oxidative stress in muscle tissue after ES

To examine the effects of oral CHM B307 treatment on oxidative stress in muscle tissue after ES, we evaluated expression levels of SOD2 in gastrocnemius muscles using IHC and biochemical analysis. As indicated in Figure 5, SOD2 expression in the Pre+ES, Post+ES, and Dual+ES mice was markedly higher than in the Sham+ES mice. Western blotting (Fig. 6Aa) revealed SOD2 expression in the treated mice was significantly greater than that in the Sham+ES mice ( $p<0.01-0.05$, Fig. 6Ab), Notably, SOD2 expression in the Dual+ES mice was significantly higher than in the Pre+ES and Post+ES mice $(p<0.05$, Fig. 6Ab).

We further examined blood ROS expression among the 4 groups through chemiluminescence analysis, because SOD2 can clear mitochondrial ROS. Figure 6Ba shows that the expression of blood ROS in the treated mice was lower than in the Sham+ES mice, and the expression of blood ROS in the Dual+ES mice was lower than in the Pre+ES and Post+ES groups. Figure 6Bb shows that quantified blood ROS expression in gastrocnemius muscle tissue of the Pre+ES, Post+ES, and Dual+ES mice was 
242 significantly lower than in the Sham+ES mice $(P<0.01-0.05$, Fig. 6Bb). Notably,

243 quantified blood ROS expression in the Dual+ES mice was significantly lower than in the 244 Pre+ES and Post+ES groups $(p<0.01)$.

\section{Effect of oral B307 treatment on muscle tissue inflammation after ES}

To examine the effects of oral CHM B307 treatment on muscle tissue inflammation after ES, we compared expressions of TNF-a and NFKB in the gastrocnemius muscles of mice after ES. As revealed by IHC staining (Fig. 7), TNF-a expression in the treated mice was markedly weaker than in the Sham+ES mice. Furthermore, as Figure 8 shows, NFKB expression in the treatment groups was obviously weaker than in the Sham+ES group.

\section{Effect of oral B307 treatment on muscle tissue damage after ES}

In mammalian cells, vinculin plays a role in muscle repair systems. Thus, we compared vinculin expression in gastrocnemius muscle tissue among the groups through IHC staining. As shown in Figure 9, vinculin expression in the treated mice was obviously stronger than in the sham-treated mice after ES. The results demonstrate that CHM B307 modulated focal adhesion structure in skeletal muscle after ES.

\section{Discussion}

261 The use of CHM as a sports supplement is becoming more common. Many agree that 262 the health benefits of plants are attributable to bioactive compounds that have 263 several physiological effects on the human body. Many CHMs are used to enhance 264 muscle strength and body mass. The purpose of this study was to provide evidence 265 that CHM B307 has considerable potential as a sports supplement. As suggested by chromatographic fingerprint analysis of CHM B307 (Fig. 2), ginseng and danshen are its main herbal ingredients. Many studies have reported that ginseng has antioxidant [23] and anti-inflammatory [24,25] properties. Others have reported that ginseng can improve alertness and fatigue resistance through cortisol stimulation [26]. Danshen can alleviate heart disease and ameliorate the effects of 
271 atherosclerosis in humans [27, 28] and rodents [29]. As suggested in a review paper [30],

272 ginseng has been used as an endurance performance enhancer. Alkaloid

273 supplementation can improve athletic performance for intense sprinting and cycling

274 exercises. Some other alkaloids such as green tea extracts can increase body mass

275 and improve muscle composition in athletes.

276 According to the results of this study, oral CHM B307 treatment can significantly

277 decrease leukocyte counts in the urine and neutrophils counts in the blood of mice

278 after ES (Fig. 4A, Ba). In blood cells, 5 types of leukocyte exist, of which neutrophils are

279 the most abundant. Neutrophils can mediate acute inflammatory tissue damage

280 because they can act against infectious microbes and produce ROS to kill invading

281 microorganisms [31]. After exhaustive exercise, neutrophil activation can result in the

282 overproduction of ROS, which contributes to muscle injury and leads to oxidative stress

$283[32,33]$. Our results indicate that mice largely increase their blood ROS production after

284 ES, but oral CHM B307 treatment can significantly decrease ROS production (Fig. 6B).

285 Nitochondrial SOD2 plays an essential role in endogenous antioxidant capacity, which

286 can inhibit oxidative stress. Our study demonstrated that oral CHM B307 treatment may

287 increase SOD2 expression in gastrocnemius muscle tissue in mice after ES (Figs. 5, 6A).

288 Oral CHM B307 treatment may increase endogenous antioxidant capacity after ES,

289 which can partially attenuate neutrophil activation and oxidative stress. The role of

290 CHM B307 supplements in reducing exercise-induced oxidative stress can enhance

291 muscle recovery and energy maintenance during intensive exercise in athletes.

292 Therefore, we believe that CHM B307 supplements could be an ideal candidate as a 293 sports supplement for enhancing muscle performance.

294 During intense exercise, lactic acid is produced in muscles and then accumulated,

295 which can lead to muscle fatigue and pain. Our findings indicate that lactic acid levels

296 in the blood of mice after ES considerably increase compared with normal mice, but

297 these levels were significantly decreased in these mice with oral CHM B307 treatment

298 (Fig. 4Bb). Though the possible role of CHM B307 supplements in reducing lactic acid is

299 unclear, we suggest that CHM B307 is an ideal candidate for alleviating muscle fatigue 
300 and pain after intensive exercise. In addition, neutrophil activation mediates

301 pathological processes and acute inflammatory tissue damage. Acute exercise may

302 activate the NFKB-TNFa inflammatory signaling pathway in rat skeletal muscles [34].

303 TNF-a has been associated with muscle inflammation that may be mediated by ROS 304 and NFKB, both of which upregulate ubiquitin/proteasome activity [35]. TNF-a and NFKB 305 play key roles in muscle inflammation after exhaustive exercise. Our results demonstrate 306 that oral CHM B307 treatment can decrease the expression of TNF-a and NFKB in 307 murine gastrocnemius muscles after ES (Figs. 7 and 8). In mammalian cells, vinculin is a 308 membrane-cytoskeletal protein involved in the linkage of adhesion molecules to actin 309 cytoskeleton [36]. Our study demonstrates that oral CHM B307 treatment can 310 effectively alleviate muscle injury in mice after ES by promoting the expression of 311 membrane-cytoskeletal vinculin protein (Fig. 9). We therefore conclude that CHM B307 312 could be an ideal sports supplement after exhaustive exercise because of oxygen debt 313 remission, NFKB-TNFa inflammatory signaling reduction, and injury recovery in muscle 314 tissue.

\section{Conclusions}

317 Our studies demonstrate that oral treatment of CHM B307 can effectively strengthen 318 muscle endurance by prolonging ES time (Fig. 3B), mitigating systemic inflammatory 319 response by reducing the number of leukocytes in urine and blood (Fig. 4A, Ba), 320 reducing muscle oxygen debt though reducing lactic acid production (Fig. 4Bb), 321 attenuating muscle oxidative stress by enhancing expression of anti-oxidative SOD2 322 protein (Figs. 5, 6A), reducing ROS production (Fig. 6B), alleviating muscle inflammation 323 by suppressing expression of inflammatory TNF-a and NFKB proteins (Figs. 7, 8), and 324 recovering muscle damage by promoting expression of membrane-cytoskeletal 325 vinculin protein (Fig. 9). Our study indicates that CHM B307 could be an ideal 326 candidate for a sports supplement that it should be taken before and after exhaustive 327 exercise because of its antifatigue, antioxidation, anti-inflammation, and antidamage 328 effects in skeletal muscles. 


\section{Abbreviations}

330 CHM: Chinese herbal medicine; ES: Exhaustive swimming; Pre+ES: Pretreatment + ES; Post+ES:

331 Posttreatment + ES; Dual+ES: Dual treatment + ES; ROS: Reactive oxygen species; ICR: Institute

332 of Cancer Research; LD50: Median lethal dose; CL: Chemiluminescence; IHC:

333 Immunohistochemistry; SOD2: Superoxide dismutase 2; TNF-a: Tumor necrosis factor alpha; NFKB:

334 Nuclear factor kappa-light-chain-enhancer of activated B cells; SEM: Standard error of the

335 mean; ANOVA: Analysis of variance

\section{Acknowledgements}

338 The authors would like to thank all subjects taking part in the trial. Also, the authors thank the 339 Sun Ten Pharmaceutical Co. Ltd. and Brion Research Institute of Taiwan, which 340 provided our experimental materials and conducted chromatographic fingerprint

341 analysis of the herbal formula B307. This manuscript was edited by Wallace Academic 342 Editing.

\section{Funding}

345 This work was supported by the funding of National Taiwan Normal University and the grants of 346 Ministry of Science and Technology, Taiwan (MOST 107-2321-B-003-001-, MOST

347 108-2321-B-003-001- and MOST 107-2320-B-003-003-MY3).

349 Availability of data and supporting materials section.

350 Please contact corresponding author for additional data.

352 Authors' contributions

353 TY, CY, YC: randomization of the protocol training of animals, and animal training assistance; KF, $354 \mathrm{CH}$ : literature review; WJ, LY, CW: molecular biology assays; CH: dissertation guidance,

355 interpretation of the data and drafted the manuscript; All authors read and approved the final 356 manuscript.

358 Authors' information 
360 TYC: Associate Professor of Department of Athletics, National Taiwan University

361 (hbeaverk@ntu.edu.tw); CYL: Associate Professor of Department of Athletics, National Taiwan

362 University (chiayinglien@ntu.edu.tw); YCT: Master of Department of Physical Education,

363 National Tsing Hua University (alex84020934@gmail.com); KFL: Professor of Department of

364 Physical Education, National Tsing Hua University (steve@mail.nd.nthu.edu.tw); CHH:

365 Postdoctoral of School of Life Science, National Taiwan Normal University

366 (jerrycarry.tw@gmail.com); WJW: Research Assistant of School of Life Science, National Taiwan

367 Normal University (efgy78@gmail.com); LYS: Research Assistant of School of Life Science,

368 National Taiwan Normal University (julia10025@gmail.com); CWL: Postdoctoral of School of Life

369 Science, National Taiwan Normal University (kumo.lu@gmail.com); CHW: Professor of School of

370 Life Science, National Taiwan Normal University (megawu@ntnu.edu.tw)

371

372 Ethics Approval and Consent to Participate

373 Ethical approval was obtained from the Institutional Animal Care and Use Committee of

374 National Taiwan Normal University (Protocol number: NTNU Animal Experiments No. 108011).

375

376 Consent for publication

377 Not applicable.

\section{Competing interests}

380 The authors declare that they have no competing interests.

\section{Publisher's Note}

383 Springer Nature remains neutral with regard to jurisdictional claims in published maps and 384 institutional affiliations.

\section{Author details}

387 ISchool of Life Science, National Taiwan Normal University, Taipei City, Taiwan. 2 Department of 388 Athletics, National Taiwan University, Taipei City, Taiwan. ${ }^{3}$ Department of Physical Education, 389 National Tsing Hua University, Hsinchu City, Taiwan 


\section{References}

393 1. Thirumalai T, Viviyan, Therasa S, et al. Intense and exhaustive exercise induce 
420 11. Sies H, Jones DP. Oxidative stress. In: Fink G, editor. Encyclopaedia of stress. San 421 Diego, CA: Elsevier; 2007. p. 45-48.

422 12. Andrade FH, Reid MB, Allen DG, et al. Effect of hydrogen peroxide and dithiothreitol 423 on contractile function of single skeletal muscle fibres from the mouse. J Physiol $424 \quad 1998,509(2): 565-75$.

425 13. Reid MB, Stokic DS, Koch SM, et al. N-acetylcysteine inhibits muscle fatigue in 426 humans. J Clin Invest 1994, 94(6): 2468-2474

427 14. Shindoh C, DiMarco A, Thomas A, et al. Effect of N-acetylcysteine on diaphragm 428 fatigue. J Appl Physiol 1990, 68(5):2107-13

429 15. Margonis K, Fatouros IG, Jamurtas AZ, et al. Oxidative stress biomarkers responses to 430 physical overtraining: implications for diagnosis. Free Radic Biol Med 2007, $431 \quad 43(6): 901-10$

432 16. Zhou W, Zeng G, Lyu C, et al. The Effect of Exhaustive Exercise on Plasma Metabolic 433 Profiles of Male and Female Rats. J Sports Sci Med 2019, 18(2):253-263.

434 17. Dos Reis IGM, Martins LEB, de Araujo GG, et al. Forced Swim Reliability for Exercise 435 Testing in Rats by a Tethered Swimming Apparatus. Front Physiol 2018, 9:1839 436 18. McArdle WD, Montoye HJ. The reliability of exhaustive swimming in the laboratory 437 rat. J Appl Physiol 1966, 21, 1431-1434.

438 19. Miao X, Xiao B, Shui S, et al: Metabolomics analysis of serum reveals the effect of 439 Danggui Buxue Tang on fatigued mice induced by exhausting physical exercise. $J$ $440 \quad$ Pharm Biomed Anal 2018, 151:301-309.

441 20. Lin CL, Wang SE, Hsu CH, et al. Oral treatment with herbal formula B307 alleviates 442 cardiac failure in aging R6/2 mice with Huntington's disease via suppressing oxidative stress, inflammation and apoptosis. Clin Interv Aging 2015, 10:1 173-1387.

21. Lien CY, Chuang TY, Hsu CH, et al. Oral treatment with the herbal formula B307 alleviates cardiac toxicity in doxorubicin- treated mice via suppressing oxidative stress, inflammation, and apoptosis. Onco Targets Ther 2015, 8:1 193-1210.

22. Li S and Chen Z. Evaluation of Antifatigue Effects of 20(S)-Ginsenoside Rg3 in Forced Swimming Mice. Indian J Pharm Sci 2018, 80(3):510-515. 
449

450

451

452

453

454

455

456

457

458

459

460

461

462

463

464

465

466

467

468

469

470

471

472

473

474

475

476

23. Chen XC, Zhu YG, Zhu LA, et al. Ginsenoside Rgl attenuates dopamine-induced apoptosis in PC12 cells by suppressing oxidative stress. Eur J Pharmacol 2003, 473(1):1-7.

24. Lee JS, Song JH, Sohn NW, et al. Inhibitory effects of ginsenoside Rbl on euroinflammation following systemic lipopolysaccharide treatment in mice. Phytother Res 2012, 27(9):1270-1276.

25. Lin WM, Zhang YM, Moldzio R, et al. Ginsenoside Rd attenuates neuroinflammation of dopaminergic cells in culture. J Neural Transm 2007, ( Suppl 72 ):105-112.

26. Ahuja A, Goswami A, Adhikari A, et al. Evaluation of effects of revital on physical performance in sportsmenle. Indian $\operatorname{Pr} 1992,45: 685-8$.

27. Xu YY, Wan RZ, Lin YP, et al. Recent advance on research and application of Salvia miltiorrhiza. Asian J Pharmacodyn Pharmacokinet 2007, 7(2):99-130.

28. Sieveking DP, Woo KS, Fung KP, et al. Chinese herbs Danshen and Gegen modulate key early atherogenic events in vitro. Int J Cardiol 2005, 105(1):40-45.

29. Tam WY, Chook P, Qiao M, et al. The efficacy and tolerability of adjunctive alternative herbal medicine (Salvia miltiorrhiza and Pueraria lobata) on vascular function and structure in coronary patients. J Altern Complement Med 2009, 15(4): 415-421.

30. Sellami $M$, Slimeni O, Pokrywka A, et al. Herbal medicine for sports: a review. J Int Soc Sports Nutr 2018 15:14.

31. Smith JA. Neutrophils, host defense, and Inflammation: a doubleedge sword. J Leukocyte Biol 1994, 56(6): 672-686.

32. Suzuki K, Sato H, Kikuchi T, et al. Capacity of circulating neutrophils to produce reactive oxygen species after exhaustive exercise. J Appl Physiol 1996, 81 (3): 1213-1222

33. Pizza FX, Peterson JM, Baas JH, et al. Neutrophils contribute to muscle injury and impair its resolution after lengthening contractions in mice. J Physiol 2005, 562(3): 899-91334. Ji LL, Gomez-Cabrera MC, Steinhafel N, et al: Acute exercise activates 
477 nuclear factor (NF)-kappaB signaling pathway in rat skeletal muscle. FASEB J 2004 $478 \quad 18(13): 1499-506$.

479 35. Thoma A, Lightfoot AP. NF-kB and Inflammatory Cytokine Signalling: Role in Skeletal $480 \quad$ Muscle Atrophy. Adv Exp Med Biol 2018, 1088:267-279.

481 36. Goldmann WH, Ingber DE. Intact vinculin protein is required for control of cell shape, 482 cell mechanics, and rac-dependent lamellipodia formation. Biochemical and 483 Biophysical Research Communications 2002, 290 (2): 749-55. 


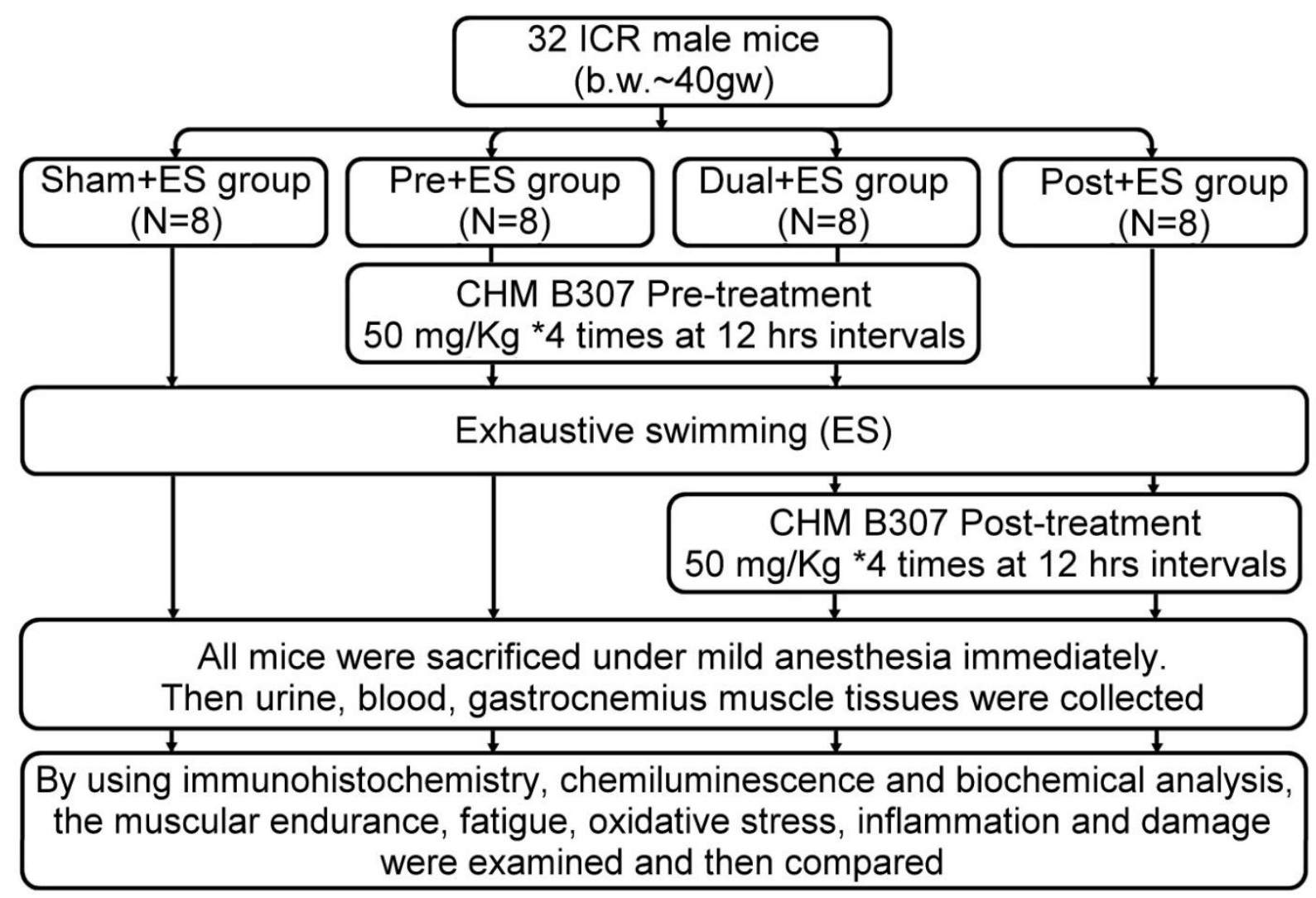

Fig. 1: Study design for assessing the effects of supplemental Chinese herbal medicine

forced swimming test. Thirty-two male ICR mice were randomly divided into 4 groups:

90 Sham + ES, CHM B307 pretreatment + ES (Pre+ES), CHM B307 posttreatment + ES

91 (Post+ES), and CHM B307 dual treatment + ES (Dual+ES). 

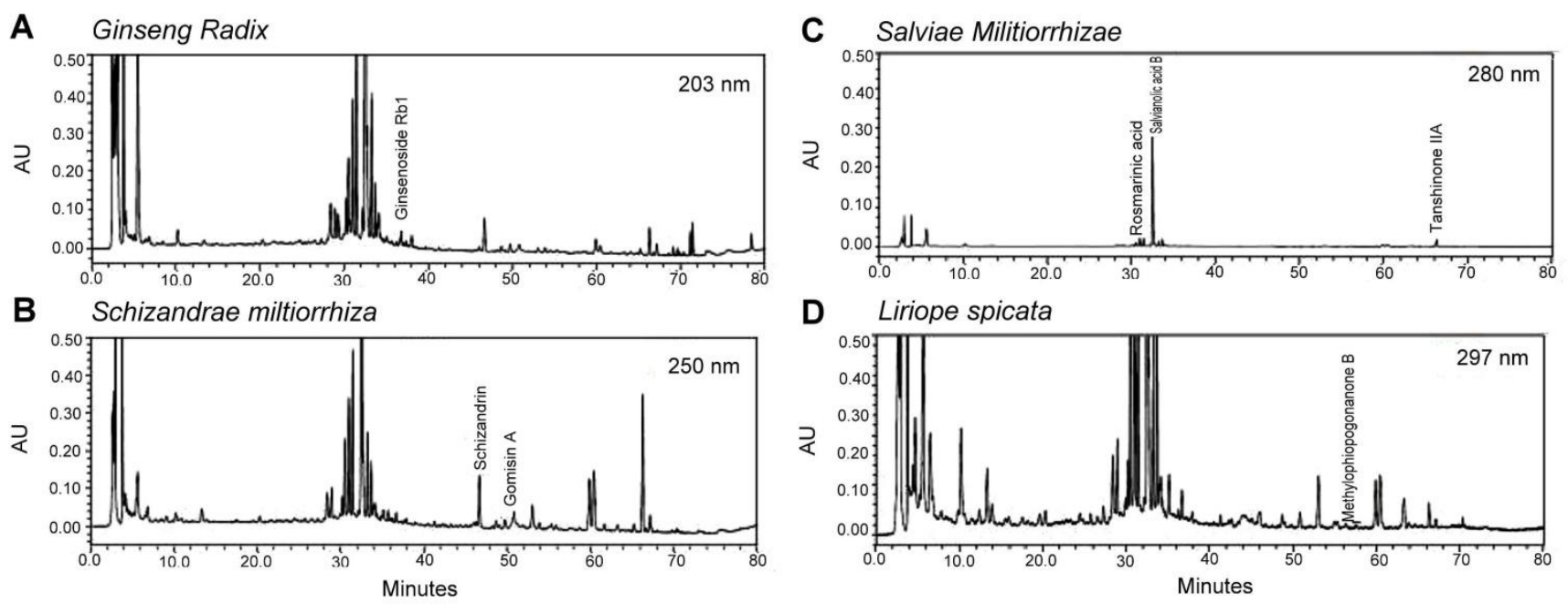

494 Fig. 2: Chromatographic fingerprints of the Chinese herbal formula B307 from

495 high-performance liquid chromatography. The peak for bioactive marker substances

496 for (A) Ginseng Radix was ginsenoside Rbl ( $\lambda=203 \mathrm{~nm}$ ); (B) peaks for Schizandrae

497 miltiorrhiza were schizandrin and gomisin A $(\lambda=250 \mathrm{~nm})$; (C) peaks for Salviae

498 Militiorrhizae Radix were rosmarinic acid, salvianolic acid B, and tanshinone IIA ( $\lambda=280$

$499 \mathrm{~nm})$; and (D) the peak for Liriope spicata was methylophiopogonanone $B(\lambda=297 \mathrm{~nm})$. 
A

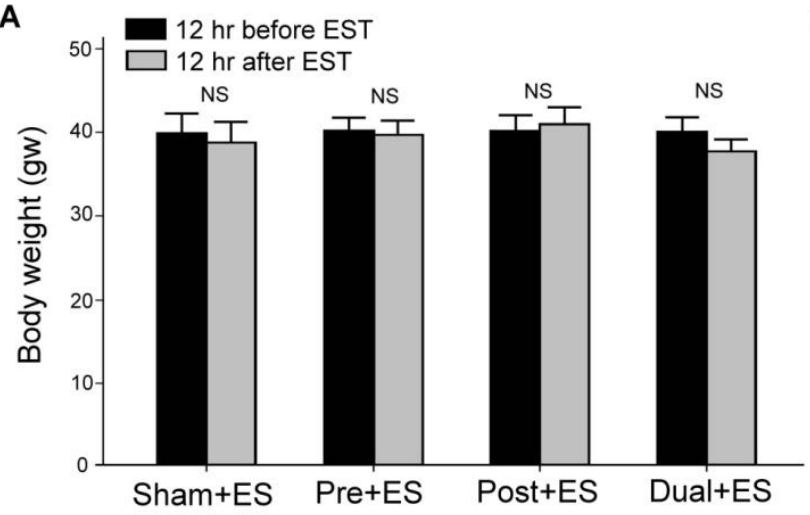

B

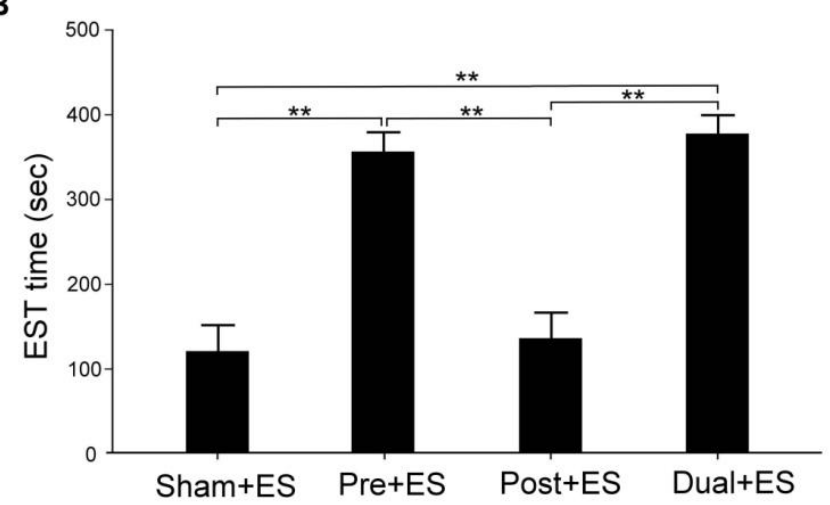

502 Fig. 3: Comparisons of the body weight and ES time of mice with and without B307

503 treatment. (A) Statistical comparison of quantified body weight $12 \mathrm{~h}$ before and after 504 the ES test. No differences (paired t test, $p>0.05$ ) were noted among the Sham+ES, 505 Pre+ES, Post+ES, and Dual+ES groups. (B) Statistical comparison of quantified ES time 506 among groups. The data indicated that the ES times of Pre+ES and Dual+ES mice was 507 significantly longer than those of Sham+ES and Post+ES mice (**P $<0.01$, one-way 508 ANOVA followed by Student-Newman-Keuls multiple comparison posttest). All data 509 are shown as mean \pm SEM. 

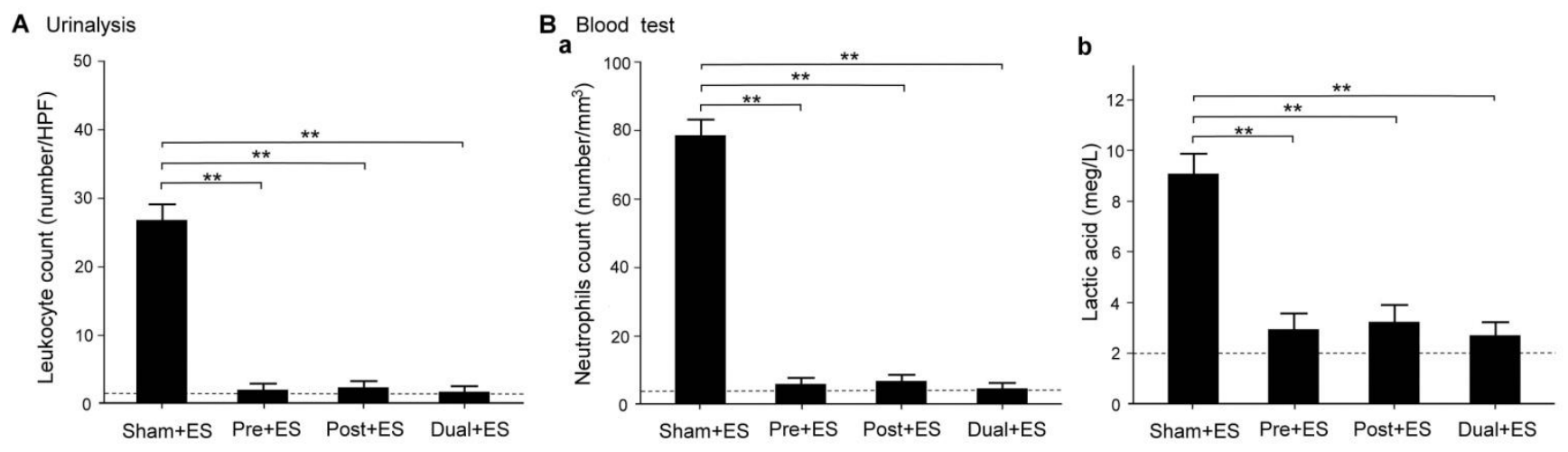

512 Fig. 4: Comparisons of urine and blood tests for mice with and without CHM B307

513 treatment. (A) Statistical comparison of quantified leukocyte counts in urine among the 514 groups. Leukocyte counts of Pre+ES, Post+ES, and Dual+ES mice were significantly lower 515 than those of Sham+ES mice $(p<0.01)$. (B) Statistical comparison of quantified 516 neutrophil counts and lactic acid in the blood of the 4 groups. Neutrophils counts and 517 lactic acid levels in the Pre+ES, Post+ES, and Dual+ES mice were significantly decreased $518(p<0.01)$. The dashed lines represent averaged values in normal mice. All data are 519 shown as mean \pm SEM $(* * P<0.01$, two-way ANOVA followed by

520 Student-Newman-Keuls multiple comparison posttest). Eight experiments were 521 conducted for each treatment. 

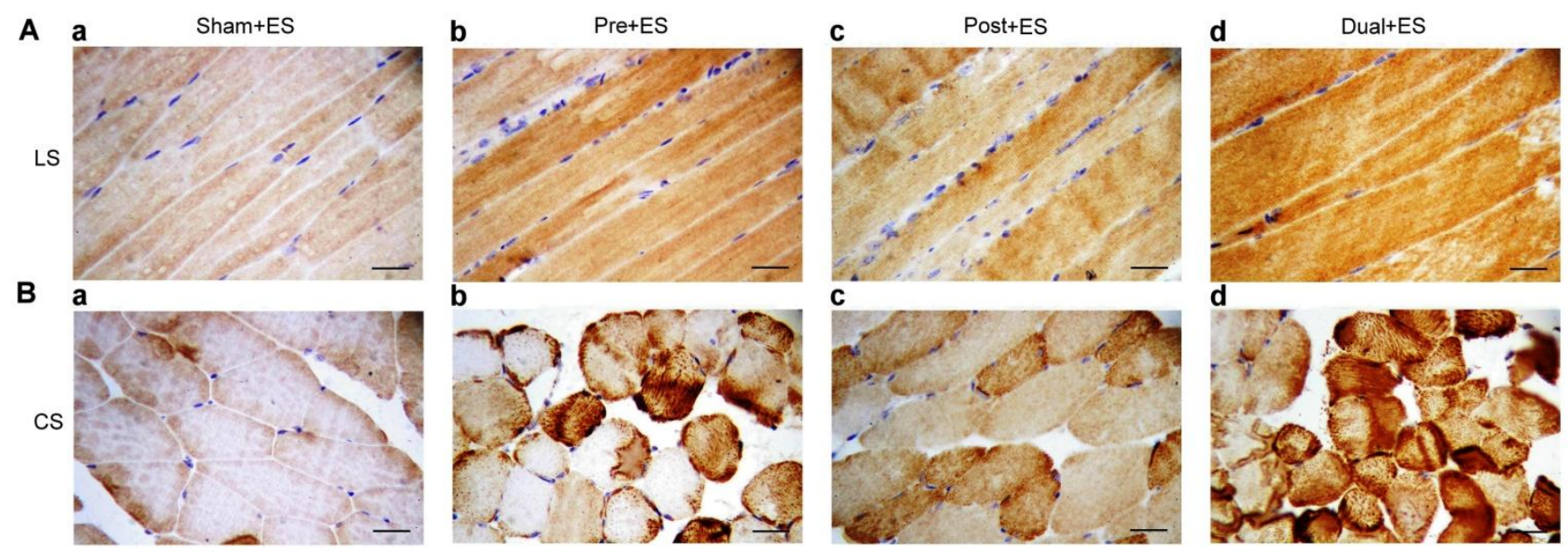

524 Fig. 5: Representative immunohistochemistry-based SOD2 expressions of 525 gastrocnemius muscle tissue between mice with and without Chinese herbal medicine 526 B307 treatment. (A) Longitudinal sections and (B) cross-sections of gastrocnemius 527 muscle tissue obtained through immunohistochemistry staining indicate that SOD2 528 expression (expressed by a deep brown color) in Pre+ES, Post+ES, and Dual+ES mice 529 (b-d) was substantially higher than in Sham+ES mice (a). Scale bar $=30 \mu \mathrm{m}$. 
Aa

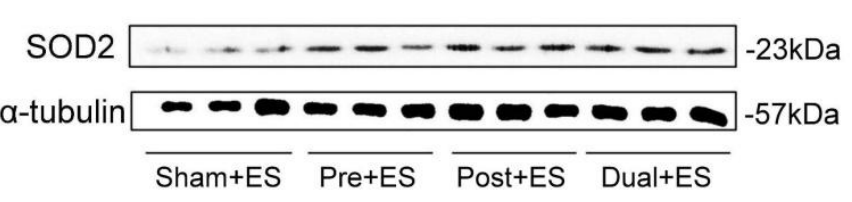

Ba

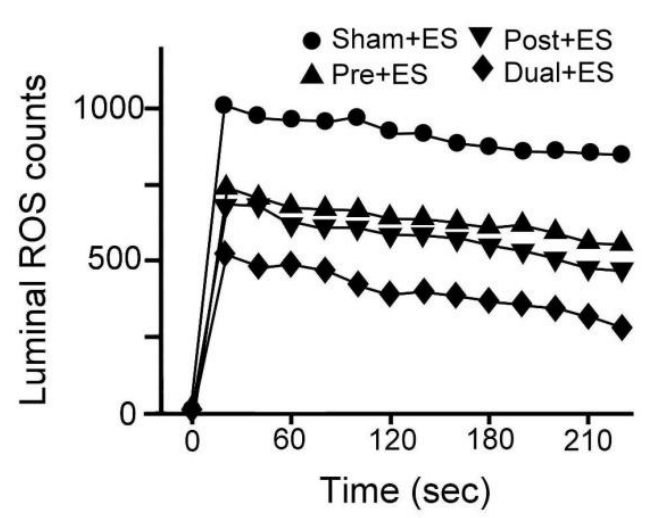

b

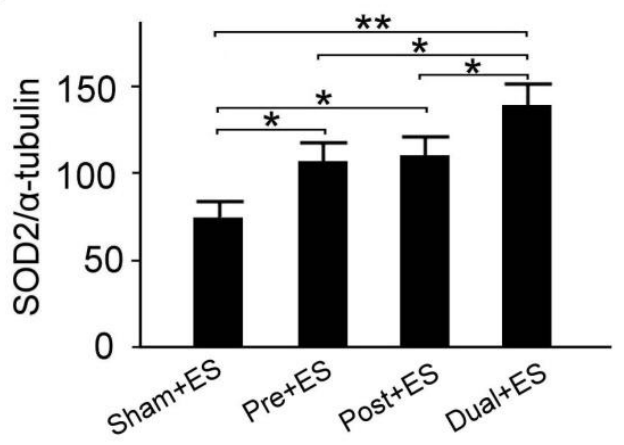

b

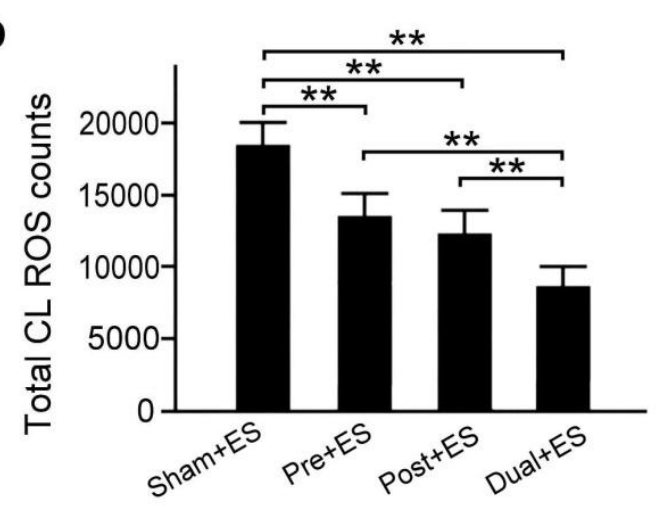

532 Fig. 6. Comparisons of muscle SOD2 and blood reactive oxygen species (ROS)

533 expression between mice with and without B307 treatment. (A) SOD2 expression of

534 gastrocnemius muscle tissue among the Sham+ES, Pre+ES, Post+ES, and Dual+ES groups

535 determined through Western blotting (a). Statistical comparison of quantified SOD2

536 expression among the groups (b). (B) Blood ROS expression among the groups

537 determined through chemiluminescence analysis (a). Statistical comparison of

538 quantified blood ROS expression among the groups (b). All data are shown as mean \pm 539 SEM $\left({ }^{* *} P<0.01,{ }^{*} P<0.05\right.$, two-way ANOVA followed by Student-Newman-Keuls 540 multiple comparison posttest). Experiments were repeated 3 times for each treatment. 


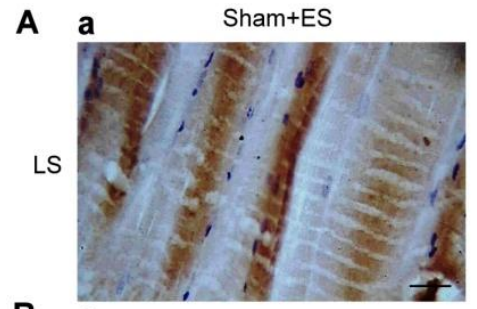

B a

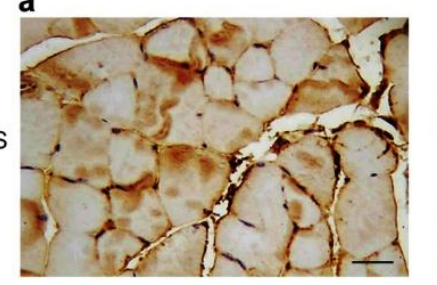

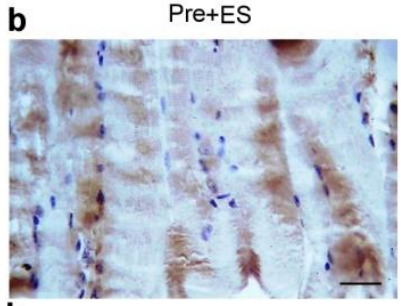

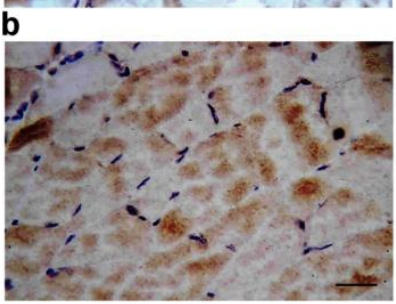

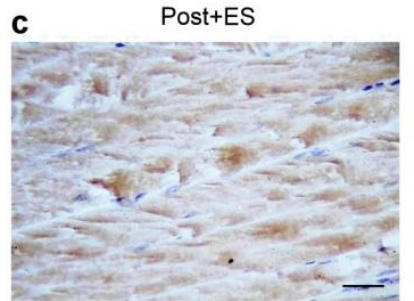

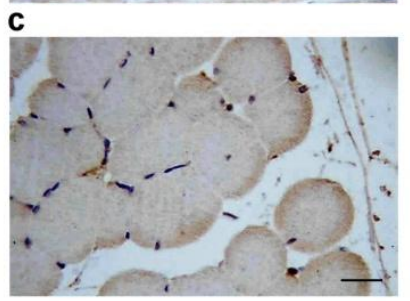

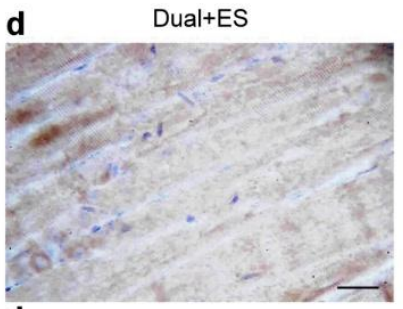

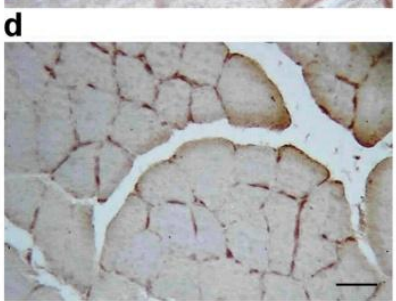

543 Fig. 7: Representative immunohistochemistry-based TNF-a expression of gastrocnemius

544 muscle tissue in response to ES between mice with and without $B 307$ treatment. (A)

545 Longitudinal sections and (B) cross-sections of gastrocnemius muscle tissue; TNF-a

546 expression (expressed by a deep brown color) in Pre+ES, Post+ES, and Dual+ES mice

$547(b-d)$ was markedly weaker than in Sham+ES mice. Scale bar $=30 \mu \mathrm{m}$. 

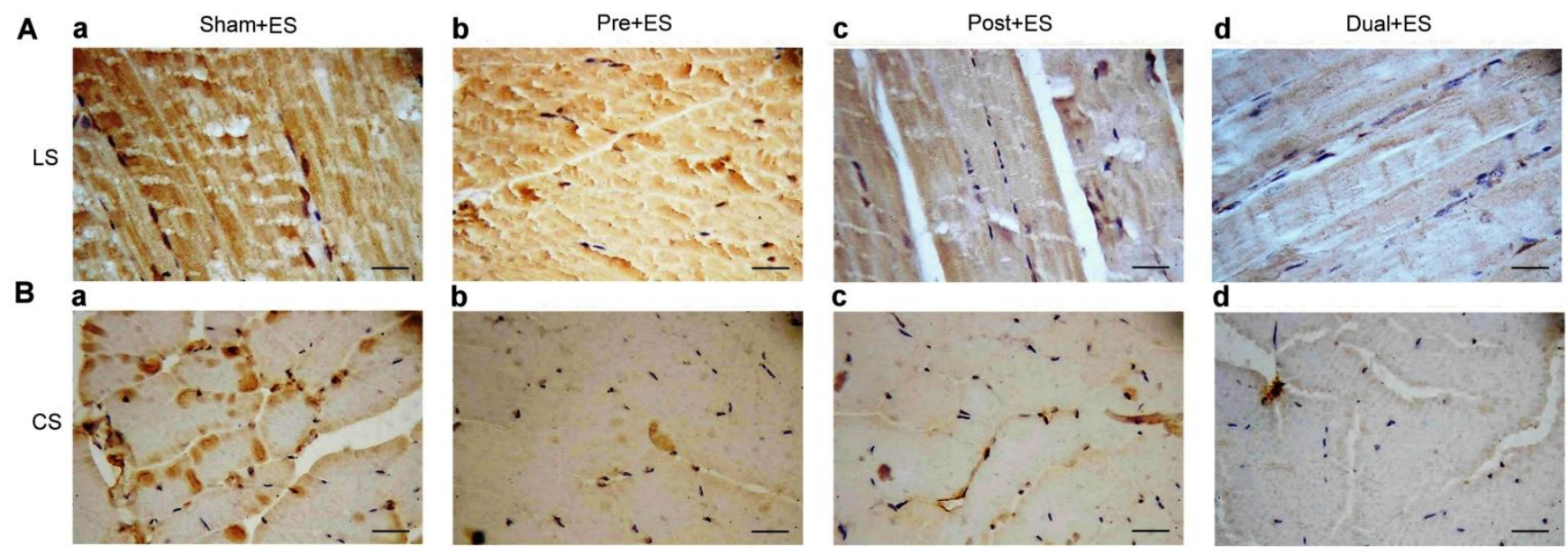

550 Fig. 8: Representative immunohistochemistry-based NFKB expression of gastrocnemius

551 muscle tissue between mice with and without B307 treatment. (A) Longitudinal sections

552 and (B) cross-sections of gastrocnemius muscle tissue through IHC staining reveal NFKB 553 expression (expressed by deep brown color) in Pre+ES, Post+ES, and Dual+ES mice (b-d) 554 was obviously weaker than in Sham+ES mice (a). Bar scale $=30 \mu \mathrm{m}$. 

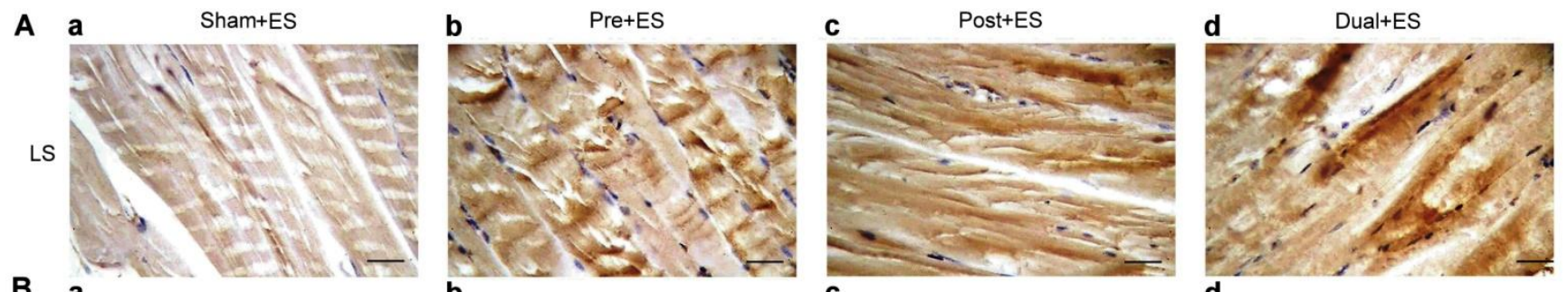

B a
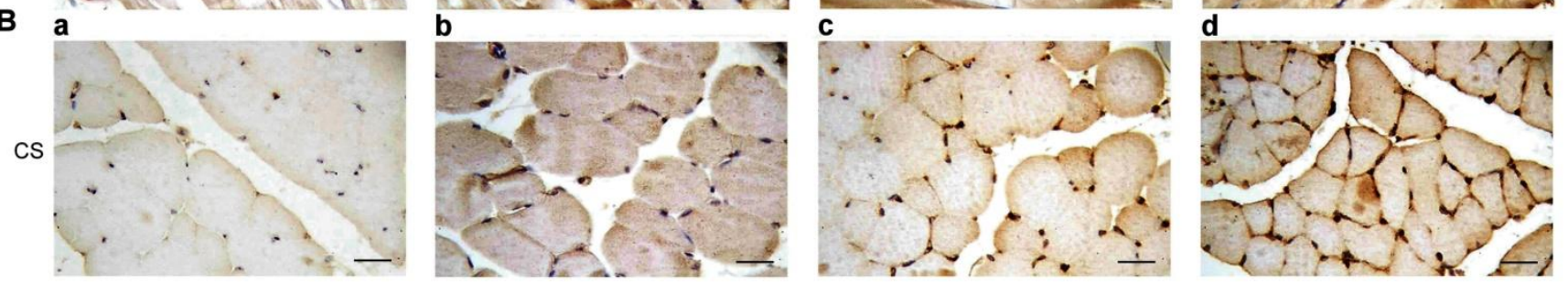

557 Fig. 9. Representative immunohistochemistry-based vinculin expression of

558 gastrocnemius muscle tissue between mice with and without B307 treatment. (A)

559 longitudinal sections and (B) cross-sections of gastrocnemius muscle tissue obtained

560 through immunohistochemical staining. The vinculin expressions (expressed by a deep

561 brown color) of Pre+ES, Post+ES, and Dual+ES mice (b-d) were markedly greater than 562 those of Sham+ES mice (a). Scale bar $=30 \mu \mathrm{m}$. 


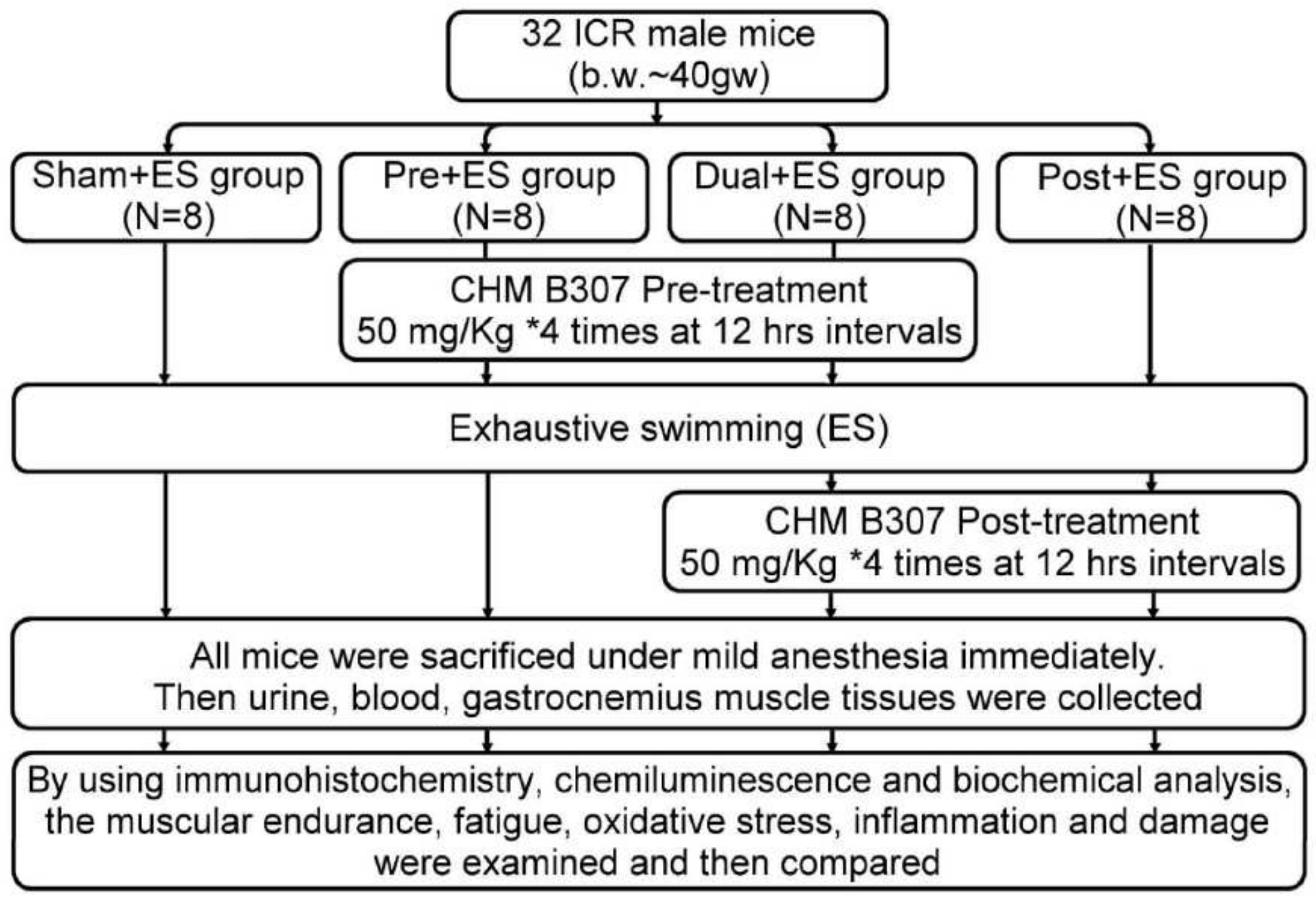

Figure 2

Study design for assessing the effects of supplemental Chinese herbal medicine B307 in ICR mice after exhaustive swimming (ES). Mice were subjected to ES under a forced swimming test. Thirty-two male ICR mice were randomly divided into 4 groups: Sham + ES, CHM B307 pretreatment + ES (Pre+ES), CHM B307 posttreatment + ES (Post+ES), and CHM B307 dual treatment + ES (Dual+ES). 


\section{A}

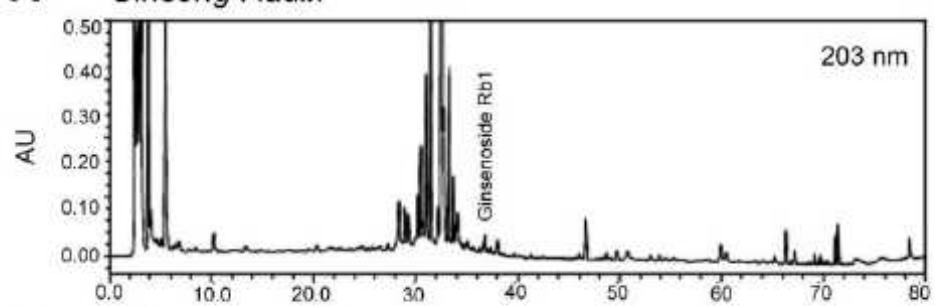

B

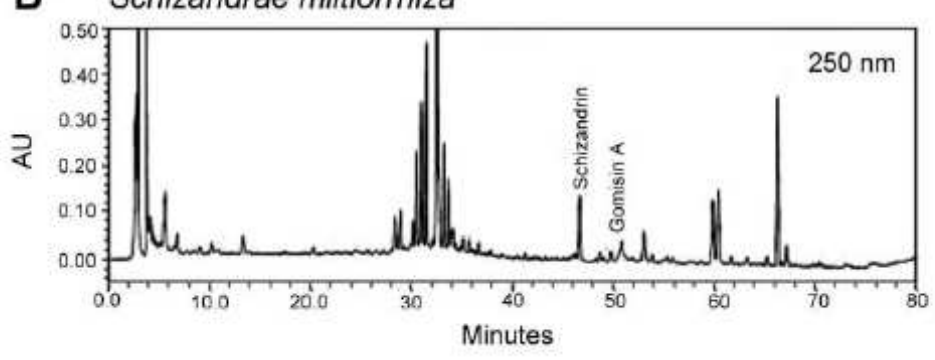

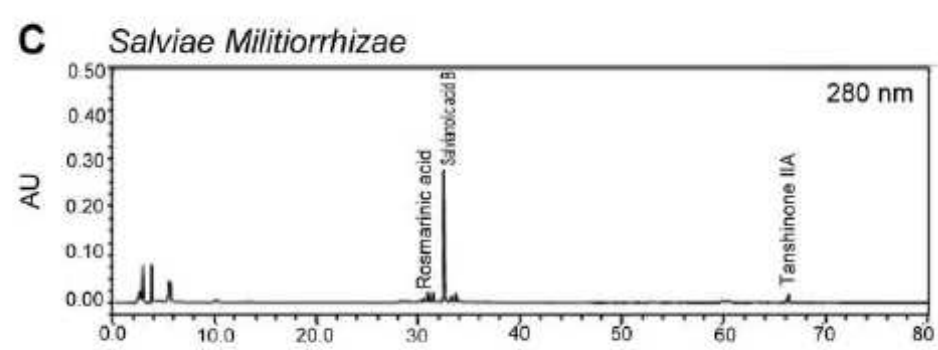

D Liriope spicata

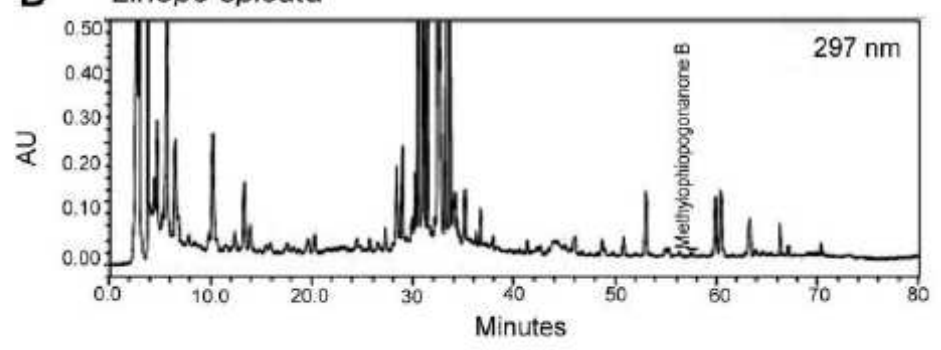

\section{Figure 4}

Chromatographic fingerprints of the Chinese herbal formula B307 from high-performance liquid chromatography. The peak for bioactive marker substances for (A) Ginseng Radix was ginsenoside Rb1( $\lambda$ $=203 \mathrm{~nm}$ ); (B) peaks for Schizandrae miltiorrhiza were schizandrin and gomisin A $(\lambda=250 \mathrm{~nm})$; (C) peaks for Salviae Militiorrhizae Radix were rosmarinic acid, salvianolic acid $B$, and tanshinone IIA $(\lambda=$ $280 \mathrm{~nm})$; and (D) the peak for Liriope spicata was methylophiopogonanone $B(\lambda=297 \mathrm{~nm})$.

A

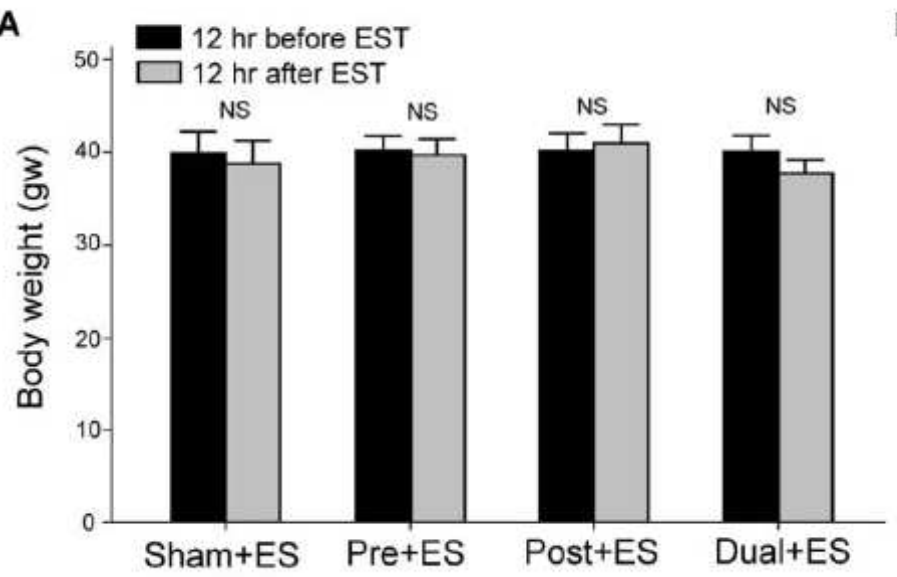

B

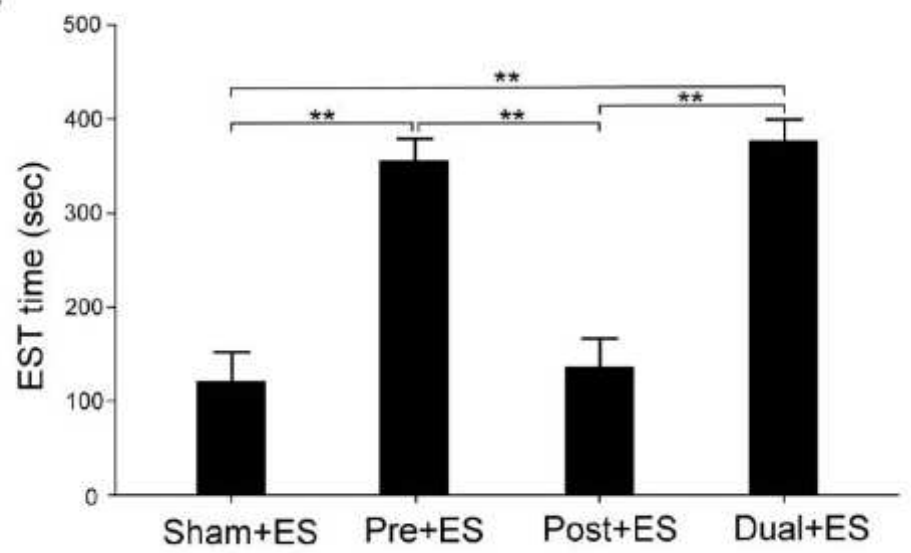

\section{Figure 6}

Comparisons of the body weight and ES time of mice with and without B307 treatment. (A) Statistical comparison of quantified body weight $12 \mathrm{~h}$ before and after the ES test. No differences (paired $t$ test, $p>$ 0.05) were noted among the Sham+ES, Pre+ES, Post+ES, and Dual+ES groups. (B) Statistical comparison of quantified ES time among groups. The data indicated that the ES times of Pre+ES and Dual+ES mice was significantly longer than those of Sham+ES and Post+ES mice ( ${ }^{\star \star P} P<0.01$, one-way ANOVA followed by Student-Newman-Keuls multiple comparison posttest). All data are shown as mean \pm SEM. 

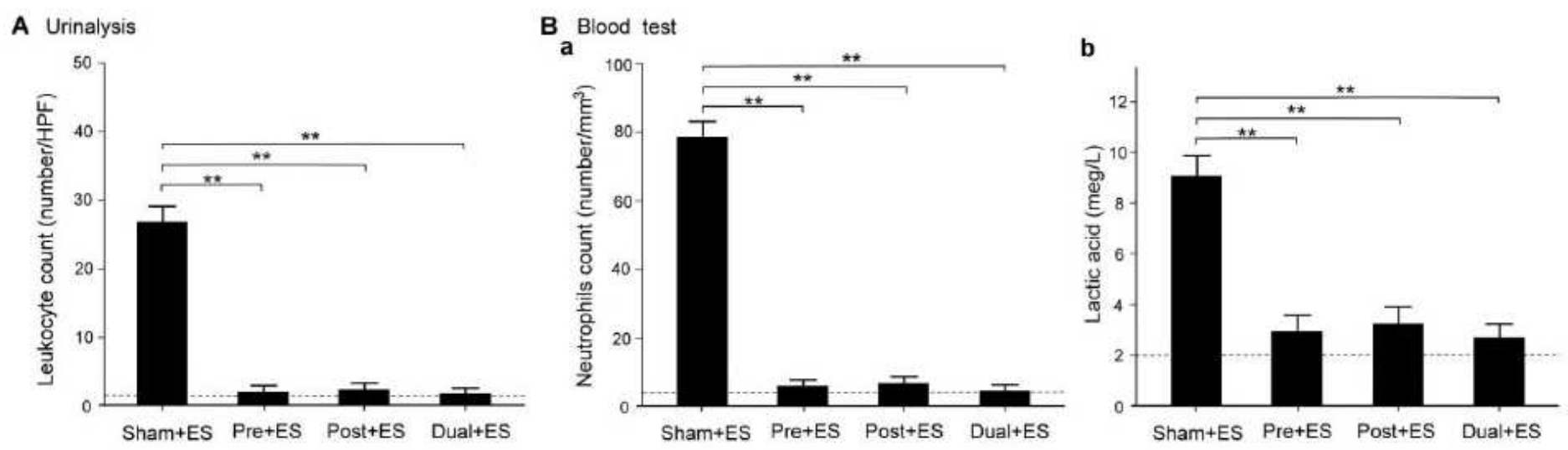

\section{Figure 8}

Comparisons of urine and blood tests for mice with and without CHM B307 treatment. (A) Statistical comparison of quantified leukocyte counts in urine among the groups. Leukocyte counts of Pre+ES, Post+ES, and Dual+ES mice were significantly lower than those of Sham+ES mice $(p<0.01)$. (B) Statistical comparison of quantified neutrophil counts and lactic acid in the blood of the 4 groups. Neutrophils counts and lactic acid levels in the Pre+ES, Post+ES, and Dual+ES mice were significantly decreased $(p<0.01)$. The dashed lines represent averaged values in normal mice. All data are shown as mean \pm SEM ( $* * P<0.01$, two-way ANOVA followed by Student-Newman-Keuls multiple comparison posttest). Eight experiments were conducted for each treatment.

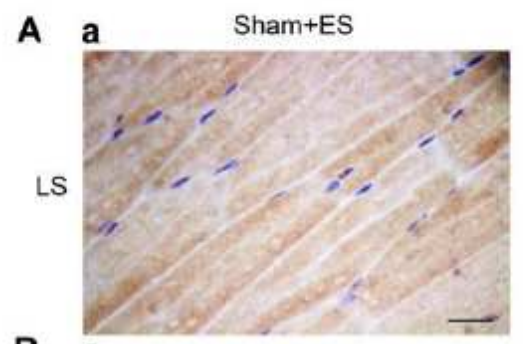

B

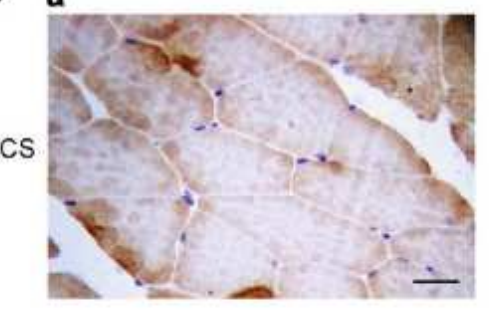

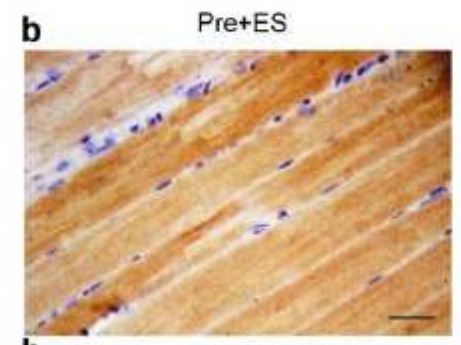

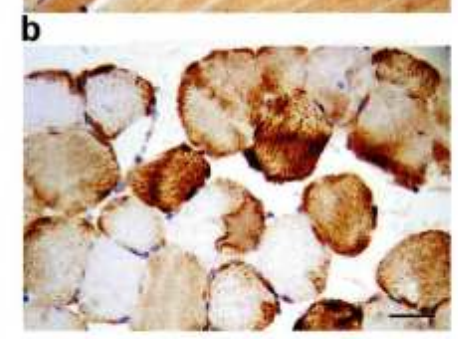

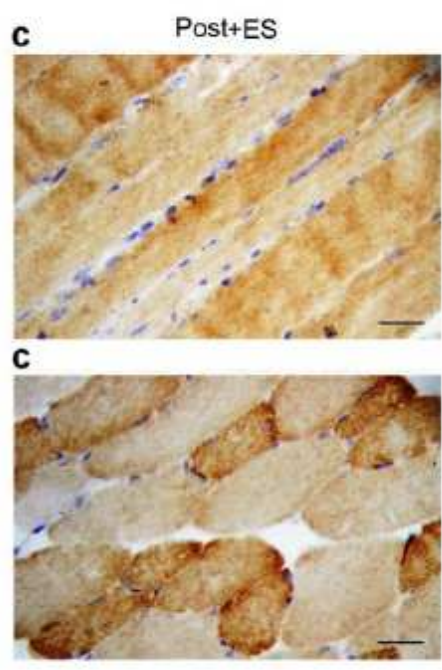

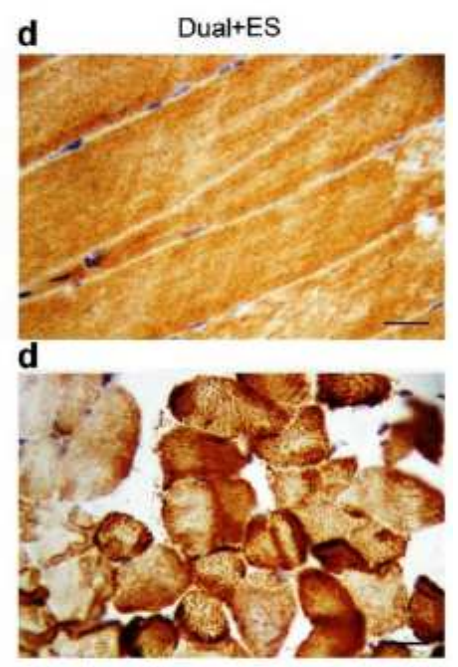

\section{Figure 10}

Representative immunohistochemistry-based SOD2 expressions of gastrocnemius muscle tissue between mice with and without Chinese herbal medicine B307 treatment. (A) Longitudinal sections and (B) cross-sections of gastrocnemius muscle tissue obtained through immunohistochemistry staining indicate that SOD2 expression (expressed by a deep brown color) in Pre+ES, Post+ES, and Dual+ES mice $(b-d)$ was substantially higher than in Sham+ES mice (a). Scale bar $=30 \mu \mathrm{m}$. 
Aa

SOD2

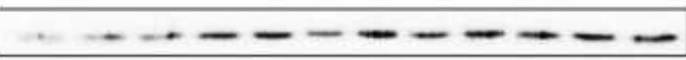

a-tubulin

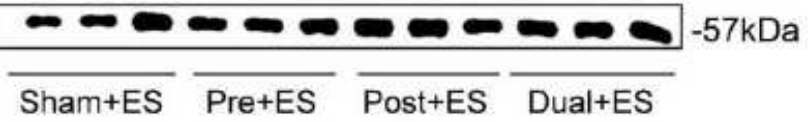

\section{$\mathrm{Ba}$}

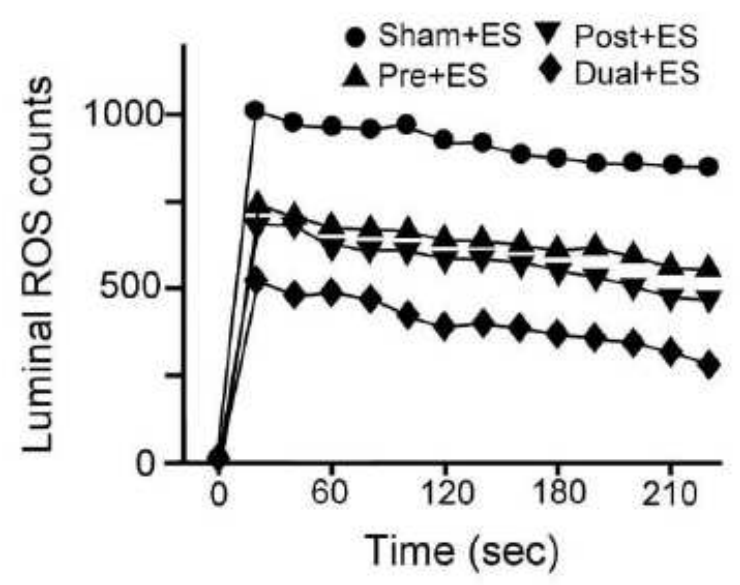

b

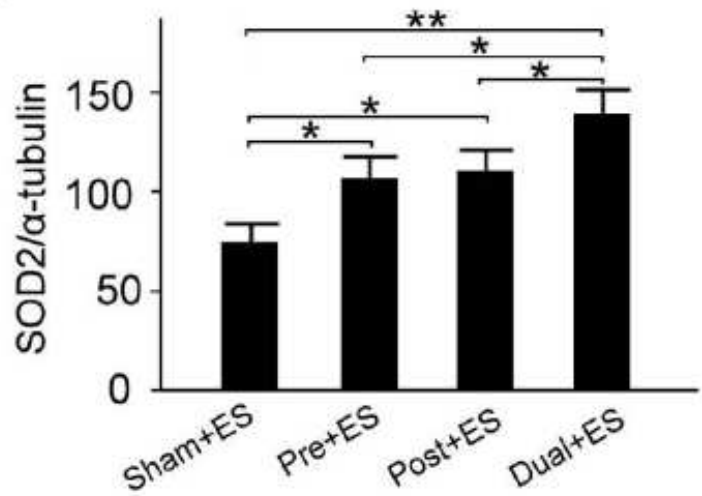

b

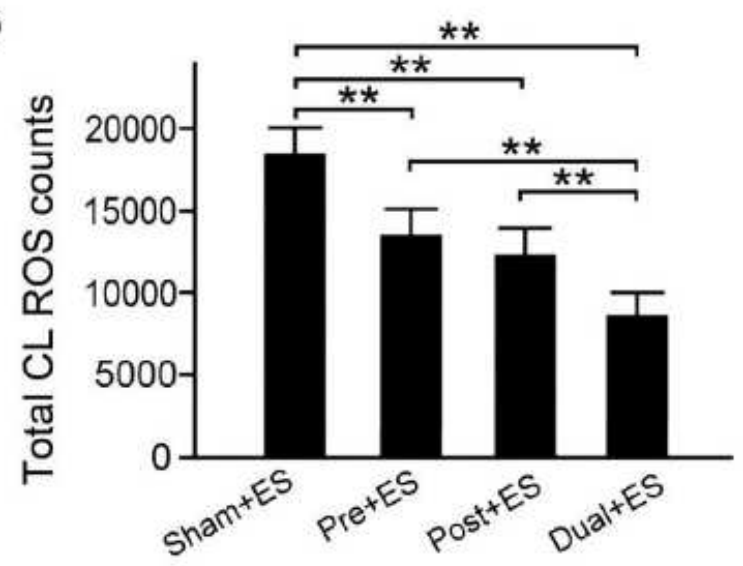

Figure 12

Comparisons of muscle SOD2 and blood reactive oxygen species (ROS) expression between mice with and without B307 treatment. (A) SOD2 expression of gastrocnemius muscle tissue among the Sham+ES, Pre+ES, Post+ES, and Dual+ES groups determined through Western blotting (a). Statistical comparison of quantified SOD2 expression among the groups (b). (B) Blood ROS expression among the groups determined through chemiluminescence analysis (a). Statistical comparison of quantified blood ROS expression among the groups (b). All data are shown as mean $\pm \mathrm{SEM}\left({ }^{\star \star} \mathrm{P}<0.01\right.$, ${ }^{\star} \mathrm{P}<0.05$, two-way ANOVA followed by Student-Newman-Keuls multiple comparison posttest). Experiments were repeated 3 times for each treatment. 


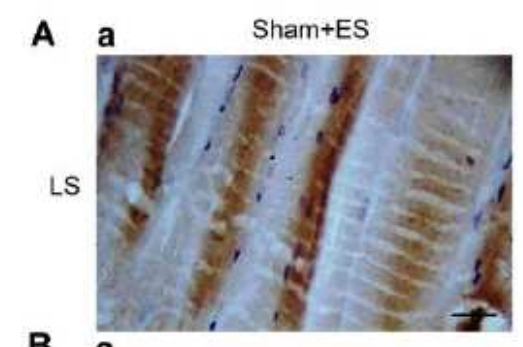

B a

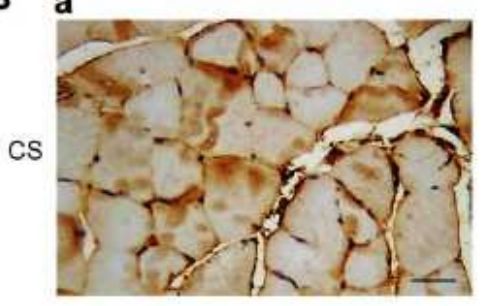

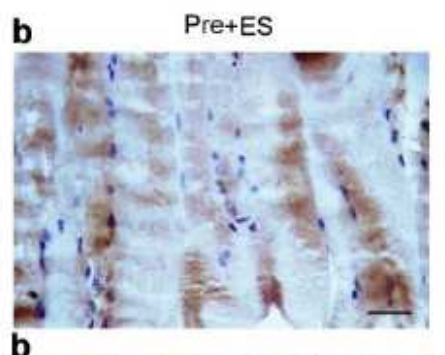

b

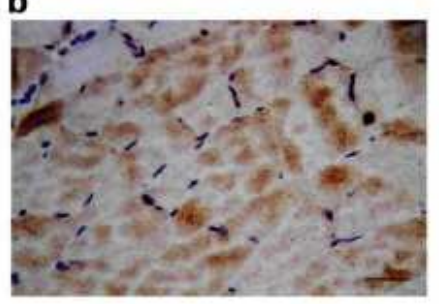

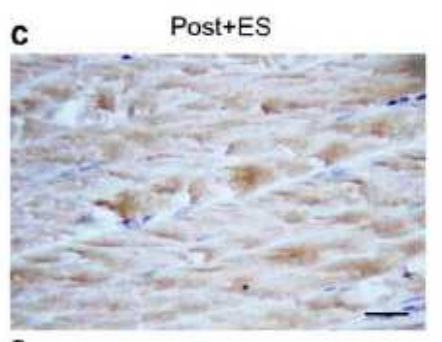

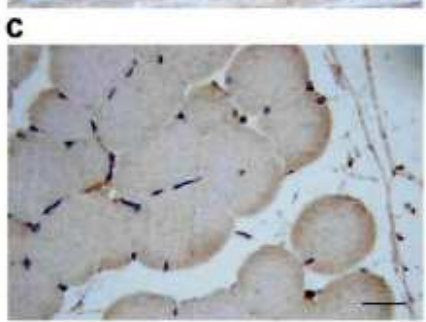

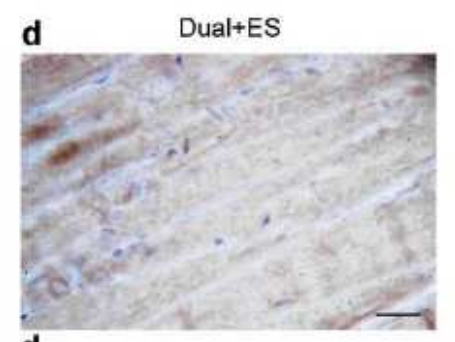

d

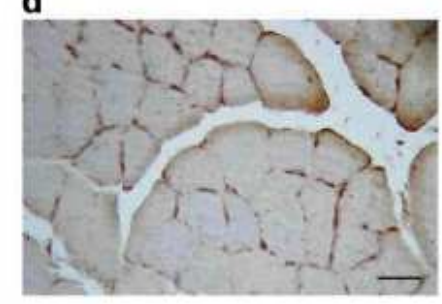

\section{Figure 13}

Representative immunohistochemistry-based TNF-a expression of gastrocnemius muscle tissue in response to ES between mice with and without B307 treatment. (A) Longitudinal sections and (B) crosssections of gastrocnemius muscle tissue; TNF-a expression (expressed by a deep brown color) in Pre+ES, Post+ES, and Dual+ES mice $(b-d)$ was markedly weaker than in Sham+ES mice. Scale bar $=30 \mu \mathrm{m}$.

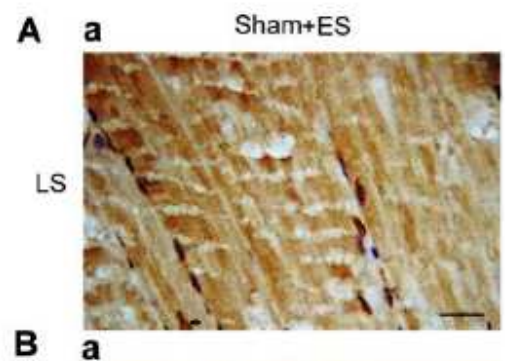

B a

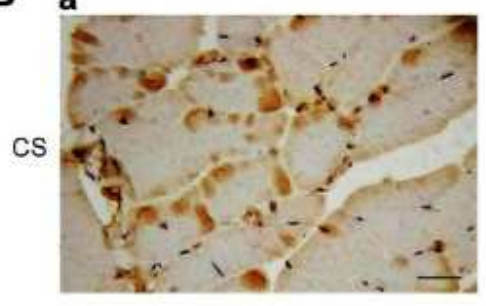

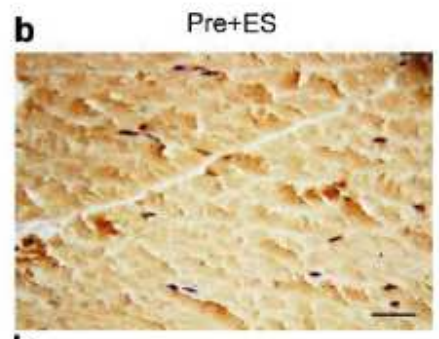

b
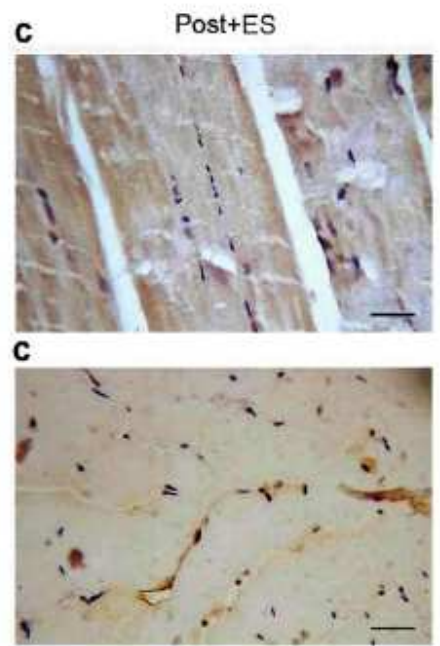

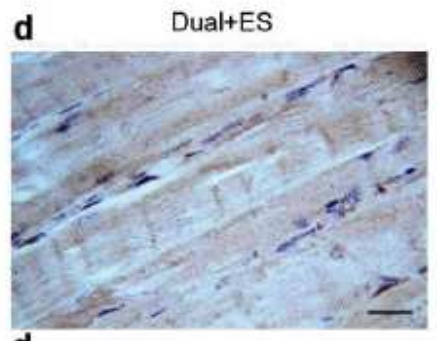

d

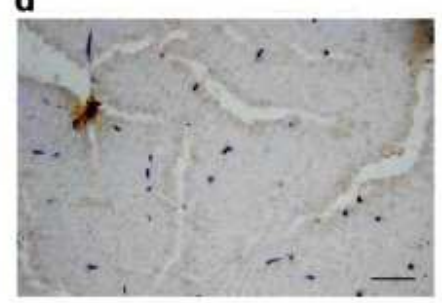

\section{Figure 16}

Representative immunohistochemistry-based NFKB expression of gastrocnemius muscle tissue between mice with and without $B 307$ treatment. (A) Longitudinal sections and (B) cross-sections of gastrocnemius muscle tissue through $\mathrm{IHC}$ staining reveal NFKB expression (expressed by deep brown color) in Pre+ES, Post+ES, and Dual+ES mice (b-d) was obviously weaker than in Sham+ES mice (a). Bar scale $=30 \mu \mathrm{m}$. 

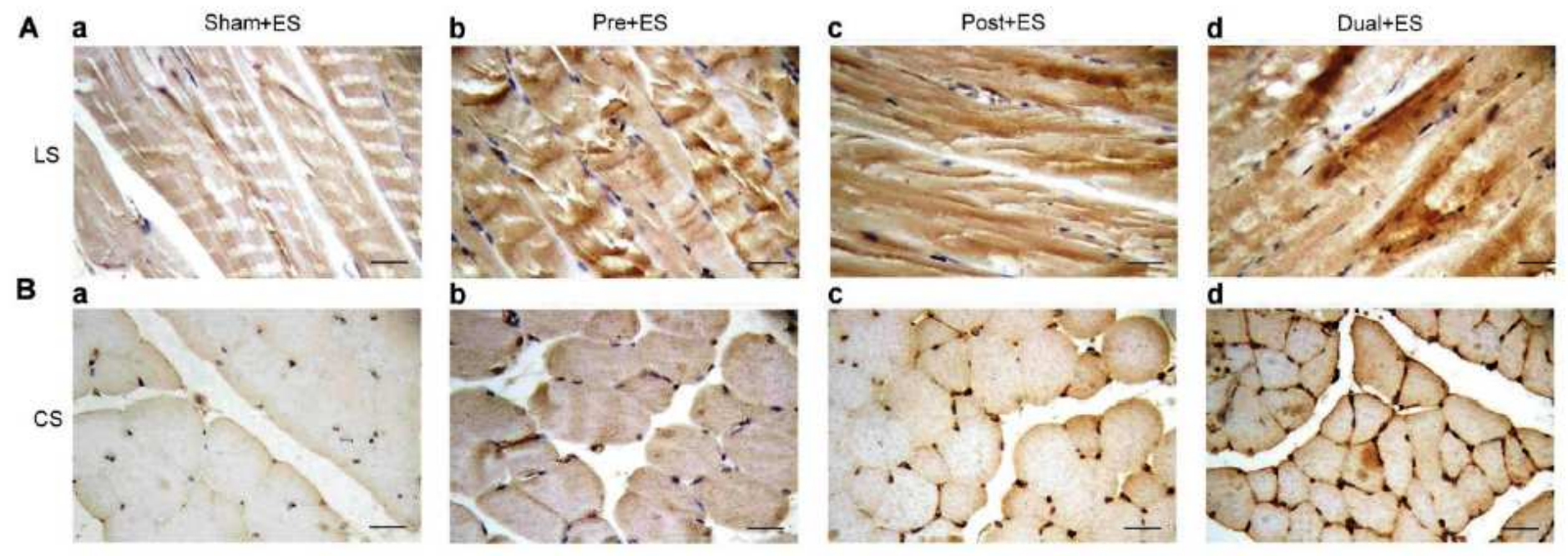

Figure 18

Representative immunohistochemistry-based vinculin expression of gastrocnemius muscle tissue between mice with and without B307 treatment. (A) longitudinal sections and (B) cross-sections of gastrocnemius muscle tissue obtained 560 through immunohistochemical staining. The vinculin expressions (expressed by a deep brown color) of Pre+ES, Post+ES, and Dual+ES mice (b-d) were markedly greater than those of Sham+ES mice (a). Scale bar $=30 \mu \mathrm{m}$. 